\title{
THE ROLE OF THE ACCRETION DISK, DUST, AND JETS IN THE IR EMISSION OF LOW-LUMINOSITY ACTIVE GALACTIC NUCLEI
}

\author{
R. E. Mason ${ }^{1}$, C. Ramos Almeida ${ }^{2,3}$, N. A. Levenson ${ }^{4}$, R. Nemmen ${ }^{5}$, and A. Alonso-Herrero ${ }^{6,7}$ \\ ${ }^{1}$ Gemini Observatory, Northern Operations Center, 670 N. A'ohoku Place, Hilo, HI 96720, USA; rmason@ gemini.edu \\ ${ }^{2}$ Instituto de Astrofísica de Canarias, C/Vía Láctea, s/n, E-38205 La Laguna, Tenerife, Spain \\ ${ }^{3}$ Departamento de Astrofísica, Universidad de La Laguna, E-38205 La Laguna, Tenerife, Spain \\ ${ }^{4}$ Gemini Observatory, Southern Operations Center, c/o AURA, Casilla 603, La Serena, Chile \\ ${ }^{5}$ NASA Goddard Space Flight Center, Greenbelt, MD 20771, USA \\ ${ }^{6}$ Instituto de Física de Cantabria, CSIC-UC, Avenida de los Castros s/n, E-39005 Santander, Spain \\ Received 2013 July 17; accepted 2013 September 25; published 2013 October 24
}

\begin{abstract}
We use recent high-resolution infrared (IR; $1-20 \mu \mathrm{m}$ ) photometry to examine the origin of the IR emission in low-luminosity active galactic nuclei (LLAGN). The data are compared with published model fits that describe the spectral energy distribution (SED) of LLAGN in terms of an advection-dominated accretion flow, truncated thin accretion disk, and jet. The truncated disk in these models is usually not luminous enough to explain the observed IR emission, and in all cases its spectral shape is much narrower than the broad IR peaks in the data. Synchrotron radiation from the jet appears to be important in very radio-loud nuclei, but the detection of strong silicate emission features in many objects indicates that dust must also contribute. We investigate this point by fitting the IR SED of NGC 3998 using dusty torus and optically thin $\left(\tau_{\text {mid-IR }} \sim 1\right)$ dust shell models. While more detailed modeling is necessary, these initial results suggest that dust may account for the nuclear mid-IR emission of many LLAGN.
\end{abstract}

Key words: accretion, accretion disks - galaxies: active - galaxies: individual (NGC 1052, NGC 3031, NGC 3998, NGC 4374, NGC 4486, NGC 4579, NGC 4594) - galaxies: nuclei - infrared: galaxies

Online-only material: color figure

\section{INTRODUCTION}

Observational and theoretical studies of low-luminosity active galactic nuclei (LLAGN; AGN with $L_{\text {bol }} \lesssim 10^{42} \mathrm{erg} \mathrm{s}^{-1}$ ) suggest that these objects differ substantially from higherluminosity AGN. At the low accretion rates typical of LLAGN, the inner part of the accretion disk is thought to take the form of a geometrically thick, optically thin, advection-dominated accretion flow (ADAF), with the standard thin accretion disk limited to a region exterior to the ADAF. If LLAGN are analogous to X-ray binaries, the radius of the transition between the inner ADAF and outer disk should increase as the accretion rate declines (Esin et al. 1997; Narayan \& McClintock 2008), and the thin disk may disappear altogether in quiescent objects. Strong winds and outflows are expected from ADAFs (e.g., Narayan \& Yi 1995), and indeed LLAGN are radio-loud compared to the general AGN population (Ho 2002; Terashima \& Wilson 2003; Nagar et al. 2005; Sikora et al. 2007).

The spectral energy distribution (SED) has been particularly useful in expanding our understanding of LLAGN. It has been instrumental, for instance, in the debate about whether LLAGN lack the "big blue bump" characteristic of the thin accretion disk in luminous AGN (e.g., Ho 1999; Maoz 2007; Eracleous et al. 2010). The high-resolution data needed to separate the nuclear and host galaxy emission have been available for some time at radio, optical/ultraviolet, and X-ray frequencies, but the first compilations of comparable infrared (IR) observations of substantial samples of LLAGN have only recently been published (Asmus et al. 2011; Mason et al. 2012, hereafter M12). The data show considerable variation among these objects. However, when considered in terms of Eddington ratio and radio loudness, they turn out to have some common characteristics. At low Eddington ratios ( $\left.\log L / L_{\text {Edd }}<-4.6\right)$, the IR-optical

\footnotetext{
7 Augusto González Linares Senior Research Fellow.
}

SEDs of radio-loud LLAGN (e.g., M87) are relatively flat (in $v L_{v}$ ) and may be dominated by synchrotron radiation. At higher $L / L_{\text {Edd }}$, the SEDs display a prominent peak in the mid-IR (MIR), and their IR-optical slopes tend to be within the range reported for higher-luminosity Seyferts.

The origin of the IR emission of LLAGN, and the process(es) responsible for the Seyfert-like IR peak in the higher- $L / L_{\mathrm{Edd}}$ objects, are currently open questions. Several mechanisms may contribute: thermal emission from dust and/or the truncated accretion disk, and nonthermal emission from the jet and/or ADAF. The nuclear IR emission of radio-quiet Seyferts and quasars comes at least partly from dust in the torus (Mor \& Netzer 2012). However, some models predict that the torus should disappear in LLAGN, either because of a lack of material reaching the central engine from the host galaxy, or because of a disk wind "switching off" at low accretion rates (Elitzur \& Shlosman 2006; Vollmer et al. 2008). The Seyfertlike IR-optical SEDs of some LLAGN, and the observation that these objects lie on the MIR/X-ray correlation established for higher-luminosity AGN, suggests that the torus could persist to fairly low luminosities (Asmus et al. 2011; M12). The strong silicate emission features observed in a number of LLAGN, though, may indicate comparatively large amounts of optically thin dust, as expected if little material is reaching or being expelled from the nucleus.

While the accretion disk emission peaks in the UV in luminous AGN (Sanders et al. 1989), that of the truncated disk is expected to shift to longer wavelengths. Consequently, the IR emission of certain LLAGN has been suggested to arise in this component (Ho 2008), and several sets of ADAF+disk+jet models predict a prominent peak from the disk between $\lambda \sim 1-10 \mu \mathrm{m}$ (e.g., Quataert et al. 1999; Ptak et al. 2004; Taam et al. 2012). Those models are barely constrained in the IR, however, because at the time of their publication, little high-resolution photometry was available. 

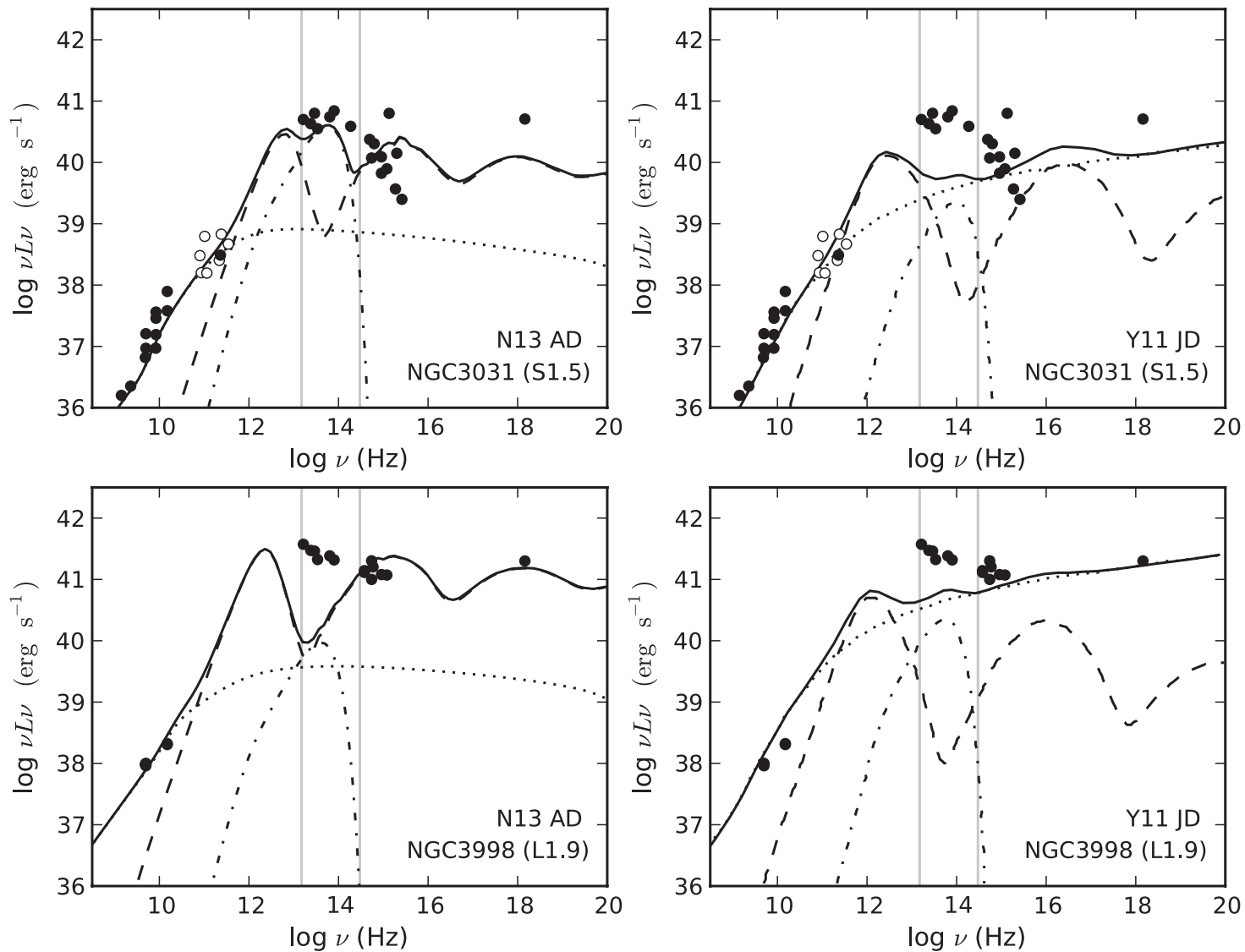

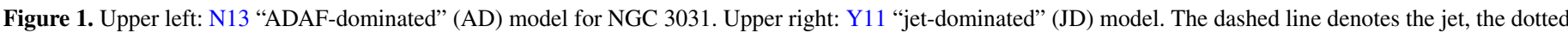


indicated by open symbols. The gray lines in each figure indicate $1 \mu \mathrm{m}$ and $20 \mu \mathrm{m}$. Lower panels: as for upper panels for NGC 3998 .

Synchrotron emission from a jet may also play a role in some objects. MIR interferometric observations of the radioloud LLAGN, Centaurus A, show two components: a resolved, disk-like structure and an unresolved core (Meisenheimer et al. 2007). While the disk component, which accounts for $20 \%-40 \%$ of the $8-13 \mu \mathrm{m}$ parsec-scale flux, is interpreted as dust heated by the AGN, the de-reddened core is well fit by a synchrotron model that turns over in the MIR.

Many LLAGN have now been modeled in terms of a jet, ADAF and truncated thin disk. The models often provide a good fit to the high-resolution radio, optical, UV, and X-ray data and have been used to estimate quantities such as mass accretion rates in LLAGN (Nemmen et al. 2013; hereafter N13). ${ }^{8}$ As a first step toward understanding the IR emission of these objects, in this paper we compare a representative set of these existing ADAF+disk+jet model fits with SEDs that include the newly available high-resolution IR data.

\section{MODEL COMPARISONS}

Of the 22 LLAGN in M12, ADAF+disk+jet models have been published for 14 (Lasota et al. 1996; Quataert et al. 1999; Gammie et al. 1999; Yuan et al. 2002; Ptak et al. 2004; Nemmen et al. 2006). We select seven for further study (Figures 1-3). The remaining galaxies' SEDs may contain a strong contribution

\footnotetext{
8 N13 differs in that (1) only 12 objects with well-sampled SEDs are chosen for detailed study; (2) jet-dominated models (Section 2) are not considered for objects with X-ray photon index $\lesssim 2$; (3) some model fits have changed, e.g., a luminous thin disk component is no longer included for NGC 4374 as the SED shows little evidence for its presence.
}

from dust heated by nuclear star formation (NGC 1097; Mason et al. 2007), are strongly affected by foreground extinction (NGC 4261; Eracleous et al. 2010), or are very sparsely sampled and/or may contain significant host galaxy emission. NGC 4258 is discussed by Wu et al. (2013).

The most extensive and uniform sets of model fits are those of N13 and Yu et al. (2011, hereafter Y11), so we use those for this comparison (see Yuan \& Cui 2005; N13 for details of the underlying models). The ADAF lies within the transition/truncation radius, $R_{\mathrm{tr}}$, outside which is found the truncated thin disk (see Figure 1 of N13). The ADAF spectrum depends on the black hole mass $M_{\mathrm{BH}}$, accretion rate $\dot{M}$, and other factors such as the viscosity parameter $\alpha$ and the fraction of the turbulent dissipation heating the electrons $\delta$. The accretion rate varies with radius, with $\dot{M}_{\text {out }}$ denoting the accretion rate at $R_{\mathrm{tr}}$. The mass loss rate in the jet, $\dot{M}_{\text {jet }}$, is required to be a reasonable fraction of the accretion rate in the ADAF's inner region (Y11), or not to exceed $\dot{M}_{\text {out }}(\mathrm{N} 13)$.

The thin disk emits locally as a blackbody and is described by $M_{\mathrm{BH}}$ (estimated from stellar velocity dispersions or gas kinematics) and the inclination angle $i$ (often constrained by radio jet observations, modeling of the broad $\mathrm{H} \alpha$ line or other measurements), along with $R_{\text {tr }}$ and $\dot{M}_{\text {out }} . R_{\text {tr }}$ and $\dot{M}_{\text {out }}$ are correlated, but the main effect of $R_{\mathrm{tr}}$ is to determine the spectral cutoff of the disk emission (smaller $R_{\text {tr }}$ implying a higherfrequency cutoff). $R_{\text {tr }}$ has little effect on the ADAF SED, as most of the ADAF emission comes from the inner region of the flow.

Y11 make initial estimates of $R_{\text {tr }}$ and $\dot{M}_{\text {out }}$, then combine these values with the other parameters $\left(M_{\mathrm{BH}}, \alpha, \delta\right.$, etc.), which are fixed, to derive the ADAF solution and SED. The disk 

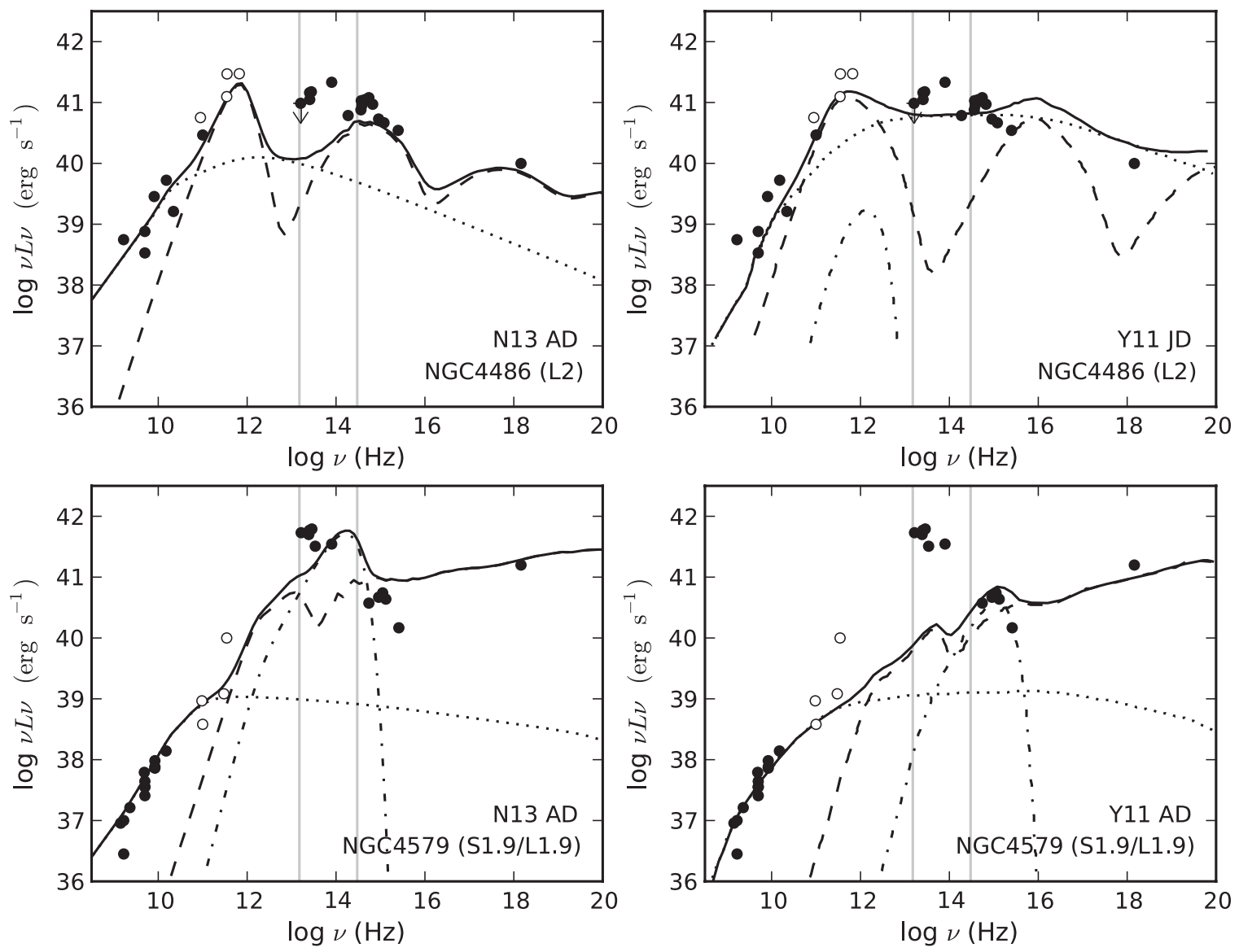

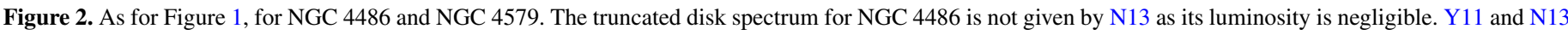
do not present JD models for NGC 4579, so both AD models are shown here.

spectrum is calculated using these initial values of $R_{\mathrm{tr}}$ and $M_{\text {out }}$, the jet SED computed, and the sum of the emission of these components compared to the observed SED. The input parameters are adjusted until a model is obtained that is roughly consistent with the data. The radio and/or X-ray data constrain $\dot{M}_{\text {out }}$, which is correlated with $R_{\text {tr }}$.

N13 first fit a jet model to the radio data, then fit the truncated disk to the optical photometry to obtain $R_{\text {tr }}$ and $\dot{M}_{\text {out }}$. The X-ray data are then used to refine the values of $R_{\mathrm{tr}}$ and $\dot{M}_{\text {out }}$, estimate $\delta$, and derive the ADAF model. The emission from the various components is then summed and compared to the overall broadband SED. Both N13 and Y11 refer to two families of models: "ADAF-dominated" (AD) and "jet-dominated" (JD), depending on which is the dominant contributor to the X-ray emission. The models are generally fit only to radio, optical, UV, and X-ray data; existing low-resolution IR points are treated as upper limits.

Figures 1-3 show the comparison between the observed SEDs (from M12 and references therein, plus Herschel farIR data for NGC 3031 from Bendo et al. 2012) and published ADAF+disk+jet models for these objects. The M12 SEDs rely heavily on those of Eracleous et al. (2010), with the addition of high-resolution IR data obtained from (near-)diffraction-limited MIR, adaptive optics and Hubble Space Telescope imaging. Outside the IR region there is a large degree of overlap between the M12 SEDs and those fitted by N13 and Y11, but they are not identical. The N13 optical/UV data use the X-ray-based extinction corrections of Eracleous et al. (2010) while those of M12 and Y11 are not corrected for extinction, and the X-ray (and some other) points chosen by each set of authors are not the same in all cases. In view of the heterogeneity of the data collections and extinction corrections, our focus here is simply to compare the model fits and SEDs in the IR region, rather than to re-examine in detail the model results over all frequencies. The reader is encouraged to consult Y11 and N13 to see the model fits to the original SEDs.

Y11 give their preferred (AD or JD) model for each nucleus, whereas N13 show both AD and JD versions unless one results in physically unreasonable parameters. Where possible, then, we show one AD and one JD model for each object. The comparison between the models and nuclear IR data shows the following.

1. The models are usually consistent with the nuclear IR data, in that they do not predict stronger emission than is observed. NGC 4579 may be an exception. Although its SED lacks the high-resolution $1-3 \mu \mathrm{m}$ data needed to make a definitive statement, the truncated disk in the N13 AD model (Figure 2) will produce too much near-IR (NIR) emission unless the spectral shape of this object is highly unusual. Similarly, Wu et al. (2013) fit an AD model to NGC 4258 and find that the disk is overluminous in the NIR. Conversely, the models never account for all of the emission over the whole $1-20 \mu \mathrm{m}$ range. There is always room for another component to contribute, particularly in the MIR.

2. The spectral shape of the truncated disk emission does not bear a good resemblance to the IR SEDs, tending to be much narrower than the broad IR peaks in the observations. It is conceivable that the disk and ADAF emission could 

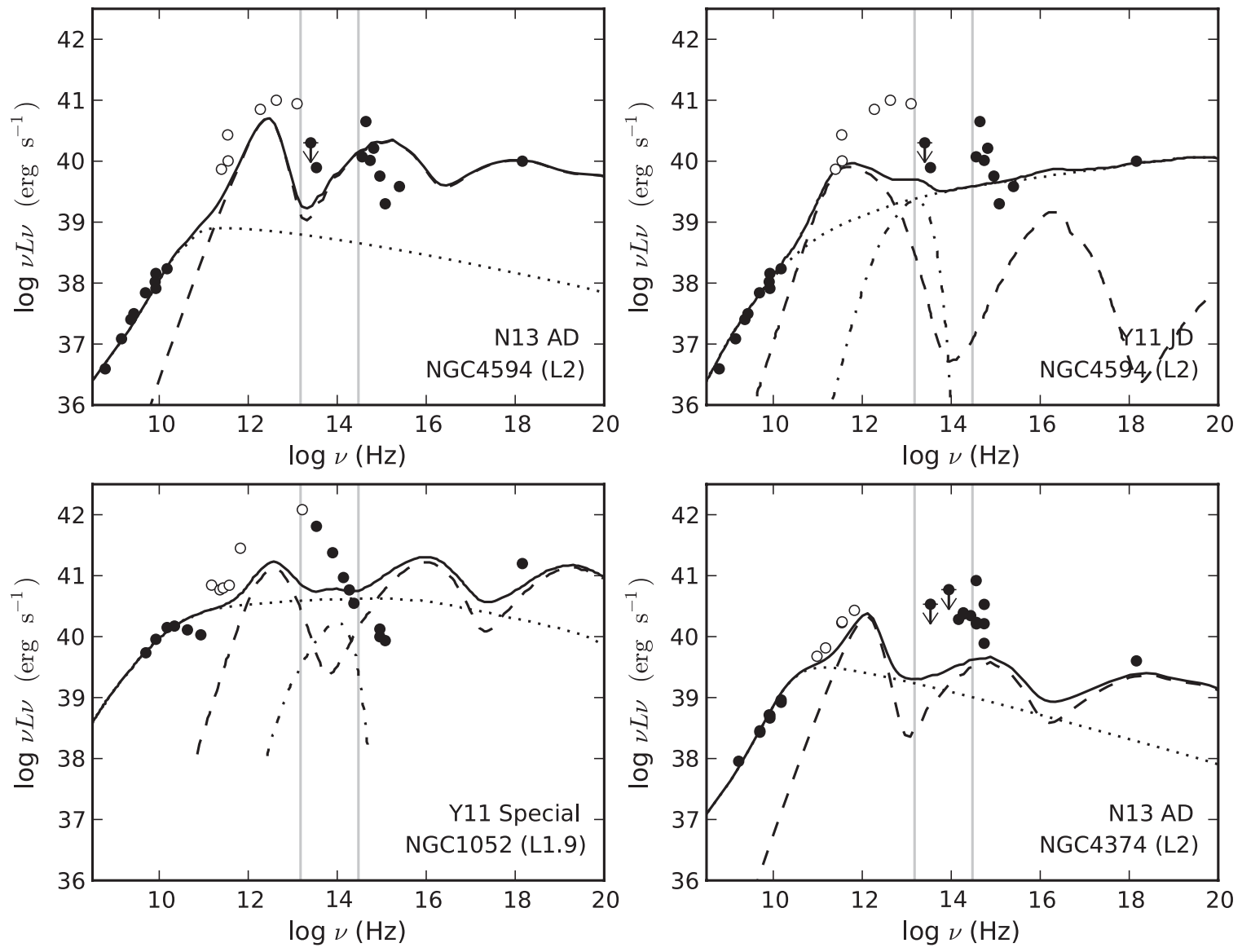


truncated disk spectrum for NGC 4374 and NGC 4594 is not given by N13 as its luminosity is negligible. The Y11 model for NGC 1052 is neither AD nor JD.

combine so as to reproduce the broad IR peak in the SEDs, but this is unlikely to happen in every object. In any case the luminosity of the truncated disk component in most of the models is much lower than the observed IR luminosity.

3. The ADAF synchrotron emission, which peaks at $v \sim$ $10^{12} \mathrm{~Hz}$, is accompanied by a Compton scattering bump at $v \sim 10^{15}-10^{16} \mathrm{~Hz}$. These two components usually bracket the nuclear IR emission.

4. The contribution of the combined ADAF+disk+jet to the observed IR emission is rather model-dependent. In NGC 3031 the Y11 JD model accounts for very little of the IR luminosity, whereas the N13 AD model predicts that most of the emission at $\lambda \sim 10 \mu \mathrm{m}$ comes from the truncated disk. The AD models tend to predict an SED whose luminosity increases sharply toward optical wavelengths, while the JD models are flatter. However, different models from the same family can also give very different results. The N13 AD model for NGC 4579 predicts strong NIR emission from the truncated disk, but the disk emission in the Y11 AD model peaks at optical wavelengths. In both models, $R_{\text {tr }}$ is consistent with constraints from the broad $\mathrm{H} \alpha$ line (Barth et al. 2001).

\section{DISCUSSION AND CONCLUSIONS}

Perhaps the most notable aspect of the comparison between the ADAF+disk+jet model fits and high-resolution SEDs is the presence of an IR "excess" above the predictions of the models. Except in one or two cases, the truncated disk is not luminous enough to account for the IR emission even over a limited range of wavelengths. The fits presented in Y11 and N13 are not unique, and it may be possible to identify ADAF models that fit the radio and X-ray emission while allowing a more luminous disk component. However, the spectral shape of the disk emission remains a poor match to the overall 1-20 $\mu \mathrm{m}$ data. We conclude that the truncated accretion disk is unlikely to be responsible for the IR "bump" in LLAGN, contrary to some recent suggestions (e.g., Ho 2008; Taam et al. 2012).

The contribution of the ADAF can be significant around $\lambda \sim 1 \mu \mathrm{m}$, but it drops off sharply toward longer wavelengths in almost all of the models. The peak frequency of the ADAF synchrotron emission depends on $M_{\mathrm{BH}}$ and $\dot{M}$ (Mahadevan 1997), and occurs at $v_{\text {peak }} \sim 10^{12} \mathrm{~Hz}$ for these LLAGN. The characteristic Compton boost parameter is such that the first peak due to the upscattering of seed synchrotron photons is typically around $10^{15}-10^{16} \mathrm{~Hz}$. The synchrotron and Compton peaks bracket the $1-20 \mu \mathrm{m}\left(\sim 10^{13}-10^{14} \mathrm{~Hz}\right)$ observations, disfavoring an ADAF origin for the nuclear IR emission.

The models do include synchrotron radiation from a jet, but the jet generally does not account for all of the IR emission. In the Y11 model for the radio galaxy NGC 1052, the jet dominates the emission at $\lambda \sim 1 \mu \mathrm{m}$ but is negligible at longer IR wavelengths. However, Fernández-Ontiveros et al. (2012) point out that the optical-IR emission of that object resembles a power law, and are able to fit the entire radio-UV SED (not corrected for extinction) with a simple self-absorbed synchrotron spectrum. This may suggest that the nuclear IR data of radio-loud objects should be included when fitting their 


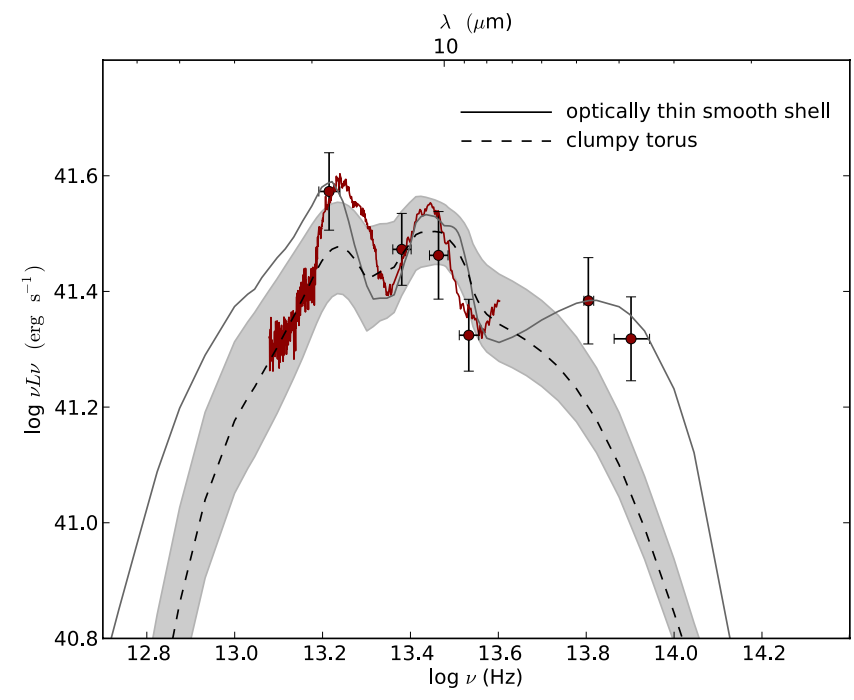

Figure 4. Clumpy torus and optically thin dust model fits for NGC 3998. The solid gray line indicates the best-fitting optically thin dust model, determined by $\chi^{2}$ minimization. The dashed line shows the clumpy torus model fit produced using the maximum a posteriori values that represent the best fit to the data, while the shaded region denotes the range of models compatible with a $68 \%$ confidence interval. The filled circles show the high-resolution photometric data points, while the red line is the Spitzer spectrum of this object $(\times 0.6)$.

(A color version of this figure is available in the online journal.)

SEDs with the ADAF+disk+jet models, potentially leading to very different derived model parameters.

A number of the LLAGN studied here, including NGC 1052, have remarkably strong silicate emission features in their spectra (M12). Many also have IR SED slopes within the range spanned by "conventional" Seyferts (e.g., Ramos Almeida et al. 2009, 2011; Prieto et al. 2010). The silicate features are an unambiguous indicator of dust, and their strength in these LLAGN suggests that they arise in dust heated by the AGN rather than in dust associated with circumstellar shells in the host galaxy (cf. Bressan et al. 2006). These observations imply that dust must contribute at some level to the nuclear IR emission of LLAGN. In Figure 4 we show two dusty model fits to the IR SED of NGC 3998: optically thin dust emission produced with the DUSTY radiative transfer code (Ivezic et al. 1999), and a clumpy torus spectrum obtained using the models of Nenkova et al. (2008) and the Bayesclumpy fitting tool (Asensio Ramos \& Ramos Almeida 2009, 2013). Clumpy torus models have been successful in fitting the SEDs of numerous Seyfert galaxies (e.g., Mason et al. 2006; Hönig \& Kishimoto 2010; Alonso-Herrero et al. 2011), while the presence of the strong silicate emission features in this object (Sturm et al. 2005, M12) suggests the use of the optically thin dust model.

In the torus model in Figure 4, the bolometric luminosity required to account for the IR emission of NGC $3998, L_{\text {bol }}=$ $1 \times 10^{42} \mathrm{erg} \mathrm{s}^{-1}$, is comparable to that estimated from the X-rays ( $L_{\text {bol }} \sim 3 \times 10^{42} \mathrm{erg} \mathrm{s}^{-1}$; assuming the bolometric correction factor of Ho 2009). The $N_{\mathrm{H}} \sim 8 \times 10^{22} \mathrm{~cm}^{-2}$ implied by the visual extinction through the clumpy torus, $A_{V}=41 \pm 4 \mathrm{mag}$, is quite close to the observed $N_{\mathrm{H}} \sim 2.3_{-1.6}^{+3.2} \times 10^{22} \mathrm{~cm}^{-2}$ (González-Martín et al. 2009), and the escape probability of an AGN-produced optical photon, $P_{\text {esc }}=0.19$, is within the range spanned by the type 1 objects in Alonso-Herrero et al. (2011). The torus models can produce a wide range of SED shapes and the photometry alone does not restrict the parameter space to a high degree. For that reason, the Spitzer spectrum was included in the fits. Although the angular resolution of the spectroscopy is a factor of $\sim 10$ lower than that of the groundbased photometry, its spectral shape matches the photometric data well. With the constraints imposed by the spectroscopy, the torus model does predict silicate features in emission, although not as strongly as observed.

The torus appears underluminous at $\lambda<5 \mu \mathrm{m}$. Given the uncertainty in the luminosity of the ADAF+disk+jet in the NIR (Section 2), it is not yet clear whether the torus model is deficient in hot dust emission, or whether the observed emission at these wavelengths contains a contribution from sources other than dust. In the ADAF+disk+jet models considered for NGC 3031, the largest contribution to the observed IR emission arises in the Y11 model around 3-4 $\mu \mathrm{m}$, where the model would account for $\sim 30 \%$ of the observed luminosity. At longer wavelengths the ADAF+disk+jet contribution would be within the photometric errors. We have subtracted the Y11 model from the data and refit the residual emission, finding only minor changes to the derived parameters $\left(L_{\mathrm{bol}}=1 \times 10^{42} \mathrm{erg} \mathrm{s}^{-1}, A_{V}=38 \pm 5 \mathrm{mag}\right.$, $\left.P_{\text {esc }}=0.30\right)$.

The basic features of the optically thin dust model are described in Sirocky et al. (2008). Briefly, the dust is distributed in a spherical shell around a broken power-law AGN spectrum, from a minimum radius corresponding to the dust sublimation temperature $(1500 \mathrm{~K})$. Dust that is optically thin in the MIR naturally produces silicate features in emission, so the Spitzer Infrared Spectrograph spectrum was not included in these fits. The best-fitting model (Figure 4) provides a reasonable description of the SED of NGC 3998, and the strength of the silicate emission features is comparable to those observed in the Spitzer spectrum. More sophisticated treatment of grain chemistry/size/structure and/or radiative transfer effects may be necessary to improve the match between the observed and modeled feature profiles (e.g., Nikutta et al. 2009; Smith et al. 2010). The $V$-band optical depth of the model, $\tau_{V}=33$, means that the AGN is heavily obscured in the optical. This is not consistent with the detection of broad $\mathrm{H} \alpha$ emission in this object (Devereux 2011). A toroidal dust distribution, not considered in this simple model, would allow direct views of the broad-line region without significantly affecting the reprocessed spectrum.

More in-depth modeling of this and other nuclei will certainly be necessary for a full understanding of the IR emission mechanisms of LLAGN. If confirmed, optically thin dust emission would be consistent with a "disappearing torus" scenario in which clouds forming in the accretion disk wind of a low-luminosity AGN do not become sufficiently optically thick to remain coherent and form an obscuring torus (Elitzur \& Shlosman 2006). Additional high-resolution NIR imaging and MIR spectroscopy would allow firmer constraints to be placed on the dusty models, and IR polarimetry (with MMT-POL, for example; Packham et al. 2012) may help disentangle the contributions of dust and jet emission to the IR luminosity of radioloud LLAGN. However, on the basis of the IR "excess" above the ADAF+disk+jet model fits examined here, the mismatch between the shape of the observed IR SED and that of the ADAF+disk+jet models, and the ability of dusty models to account for the IR SED, silicate emission and other characteristics of NGC 3998, we suggest that dust contributes strongly to the emission of many low-luminosity AGN between $\sim 1-30 \mu \mathrm{m}$.

We thank the referee for a useful report that helped improve this work. Supported by the Gemini Observatory, operated by the Association of Universities for Research in Astronomy, Inc., on behalf of the international Gemini partnership of 
Argentina, Australia, Brazil, Canada, Chile, and the USA. A.A.-H. acknowledges support from the Spanish Plan Nacional de Astronomía y Astrofísica under grant AYA2009-05705-E, CRA from PN-AYA2010-21887-C04.04. R.N. was supported by an appointment to the NASA Postdoctoral Program at Goddard Space Flight Center, administered by Oak Ridge Associated Universities through a contract with NASA.

\section{REFERENCES}

Alonso-Herrero, A., Ramos Almeida, C., Mason, R., et al. 2011, ApJ, 736, 82 Asensio Ramos, A., \& Ramos Almeida, C. 2009, ApJ, 696, 2075

Asensio Ramos, A., \& Ramos Almeida, C. 2013, MNRAS, 428, 195

Asmus, D., Gandhi, P., Smette, A., Hönig, S. F., \& Duschl, W. J. 2011, A\&A, 536, A36

Barth, A. J., Ho, L. C., Filippenko, A. V., Rix, H.-W., \& Sargent, W. L. W. 2001, ApJ, 546, 205

Bendo, G. J., Boselli, A., Dariush, A., et al. 2012, MNRAS, 419, 1833

Bressan, A., Panuzzo, P., Buson, L., et al. 2006, ApJL, 639, L55

Devereux, N. 2011, ApJ, 727, 93

Elitzur, M., \& Shlosman, I. 2006, ApJL, 648, L101

Eracleous, M., Hwang, J. A., \& Flohic, H. M. L. G. 2010, ApJ, 711, 796

Esin, A. A., McClintock, J. E., \& Narayan, R. 1997, ApJ, 489, 865

Fernández-Ontiveros, J. A., Prieto, M. A., Acosta-Pulido, J. A., \& Montes, M. 2012, JPhCS, 372, 012006

Gammie, C. F., Narayan, R., \& Blandford, R. 1999, ApJ, 516, 177

González-Martín, O., Masegosa, J., Márquez, I., \& Guainazzi, M. 2009, ApJ, 704, 1570

Ho, L. C. 1999 , ApJ, 516, 672

Ho, L. C. 2002, ApJ, 564, 120

Ho, L. C. 2008, ARA\&A, 46, 475

Ho, L. C. 2009, ApJ, 699, 626

Hönig, S. F., \& Kishimoto, M. 2010, A\&A, 523, A27

Ivezic, Z., Nenkova, M., \& Elitzur, M. 1999, arXiv:astro-ph/9910475

Lasota, J.-P., Abramowicz, M. A., Chen, X., et al. 1996, ApJ, 462, 142

Mahadevan, R. 1997, ApJ, 477, 585
Maoz, D. 2007, MNRAS, 377, 1696

Mason, R. E., Geballe, T. R., Packham, C., et al. 2006, ApJ, 640, 612

Mason, R. E., Levenson, N. A., Packham, C., et al. 2007, ApJ, 659, 241

Mason, R. E., Lopez-Rodriguez, E., Packham, C., et al. 2012, AJ, 144, 11

Meisenheimer, K., Tristram, K. R. W., Jaffe, W., et al. 2007, A\&A, 471, 453

Mor, R., \& Netzer, H. 2012, MNRAS, 420, 526

Nagar, N. M., Falcke, H., \& Wilson, A. S. 2005, A\&A, 435, 521

Narayan, R., \& McClintock, J. E. 2008, NewAR, 51, 733

Narayan, R., \& Yi, I. 1995, ApJ, 444, 231

Nemmen, R. S., Storchi-Bergmann, T., \& Eracleous, M. 2013, MNRAS, submitted

Nemmen, R. S., Storchi-Bergmann, T., Yuan, F., et al. 2006, ApJ, 643, 652

Nenkova, M., Sirocky, M. M., Ivezić, Ž., \& Elitzur, M. 2008, ApJ, 685, 147

Nikutta, R., Elitzur, M., \& Lacy, M. 2009, ApJ, 707, 1550

Packham, C., Jones, T. J., Warner, C., et al. 2012, Proc. SPIE, 8446, 3

Prieto, M. A., Reunanen, J., Tristram, K. R. W., et al. 2010, MNRAS, 402,724

Ptak, A., Terashima, Y., Ho, L. C., \& Quataert, E. 2004, ApJ, 606, 173

Quataert, E., Di Matteo, T., Narayan, R., \& Ho, L. C. 1999, ApJL, 525, L89

Ramos Almeida, C., Levenson, N. A., Alonso-Herrero, A., et al. 2011, ApJ, 731, 92

Ramos Almeida, C., Levenson, N. A., Rodríguez Espinosa, J. M., et al. 2009, ApJ, 702, 1127

Sanders, D. B., Phinney, E. S., Neugebauer, G., Soifer, B. T., \& Matthews, K. 1989, ApJ, 347, 29

Sikora, M., Stawarz, Ł., \& Lasota, J.-P. 2007, ApJ, 658, 815

Sirocky, M. M., Levenson, N. A., Elitzur, M., Spoon, H. W. W., \& Armus, L. 2008, ApJ, 678, 729

Smith, H. A., Li, A., Li, M. P., et al. 2010, ApJ, 716, 490

Sturm, E., Schweitzer, M., Lutz, D., et al. 2005, ApJL, 629, L21

Taam, R. E., Liu, B. F., Yuan, W., \& Qiao, E. 2012, ApJ, 759, 65

Terashima, Y., \& Wilson, A. S. 2003, ApJ, 583, 145

Vollmer, B., Beckert, T., \& Davies, R. I. 2008, A\&A, 491, 441

Wu, Q., Yan, H., \& Yi, Z. 2013, arXiv:1305.0072

Yu, Z., Yuan, F., \& Ho, L. C. 2011, ApJ, 726, 87

Yuan, F., \& Cui, W. 2005, ApJ, 629, 408

Yuan, F., Markoff, S., Falcke, H., \& Biermann, P. L. 2002, A\&A, 391, 139 